\title{
Fractional rotational diffusion of rigid dipoles in an asymmetrical double-well potential
}

\author{
William T. Coffey, ${ }^{1}$ Yuri P. Kalmykov, ${ }^{2}$ Sergey V. Titov, ${ }^{3}$ and Jagdish K. Vij ${ }^{1}$ \\ ${ }^{1}$ Department of Electronic and Electrical Engineering, Trinity College, Dublin 2, Ireland \\ ${ }^{2}$ Groupe de Physique Moléculaire, MEPS, Université de Perpignan, 52 Avenue Paul Alduy, 66860 Perpignan Cedex, France \\ ${ }^{3}$ Institute of Radio Engineering and Electronics of the Russian Academy of Sciences, Fryazino, Moscow Region,
} 141190, Russian Federation

(Received 1 March 2005; published 12 July 2005)

\begin{abstract}
The longitudinal and transverse components of the complex dielectric susceptibility tensor of an assembly of dipolar molecules rotating in an asymmetric double-well potential are evaluated using a fractional rotational diffusion equation (based on the diffusion limit of a fractal time random walk) for the distribution function of orientations of the molecules on the surface of the unit sphere. The calculation is the fractional analog of the Debye theory of orientational relaxation in the presence of external and mean field potentials (excluding inertial effects). Exact and approximate (based on the exponential separation for normal diffusion of the time scales of the intrawell and overbarrier relaxation processes associated with the bistable potential) solutions for the dielectric dispersion and absorption spectra are obtained. It is shown that a knowledge of the characteristic relaxation times for normal rotational diffusion is sufficient to predict accurately the anomalous dielectric relaxation behavior of the system for all time scales of interest. The model explains the anomalous (Cole-Colelike) relaxation of complex dipolar systems, where the anomalous exponent differs from unity (corresponding to the normal dielectric relaxation), i.e., the relaxation process is characterized by a broad distribution of relaxation times (e.g., in glass-forming liquids).
\end{abstract}

DOI: 10.1103/PhysRevE.72.011103

PACS number(s): 05.40.Jc, 77.22.-d, 05.45.Df

\section{INTRODUCTION}

The discrete orientation or Debye-Fröhlich model of the relaxation of an assembly of dipoles in a crystalline field in its most simple form, namely, an asymmetric bistable potential with the escape time over the barrier (that is the greatest relaxation time) given by the Arrhenius law, has often been used to model relaxation processes in solids, amorphous materials, glass forming liquids, etc. [1,2]. The model is usually analyzed via a very simple rate equation approach $[3,4]$. The disadvantage of such an approach since it uses the Arrhenius law is that it is inherently assumed [5,6] that the MaxwellBoltzmann distribution obtains everywhere even in the vicinity of the barriers. Thus the prefactor of the greatest relaxation time is independent of the dissipative coupling to the heat bath. Moreover, it is not directly apparent (again since the Maxwell-Boltzmann distribution is assumed to obtain everywhere) how the discrete orientation model fits into the general theory of stochastic processes in terms of kinetic and stochastic differential equations as formulated by Boltzmann, Einstein, Smoluchowski, Langevin, and Kramers [5-7] where the dissipative coupling is taken account of via a particular Stosszahlansatz. Consequently, it is impossible in the discrete orientation approach to include the dissipative coupling to the bath (meaning interalia that the MaxwellBoltzmann distribution does not hold in the vicinity of the barrier) in the dynamics of the particles in the asymmetric potential neither is it possible to consider the relaxation processes in the wells of the potential. The foregoing difficulties may, however, be overcome by considering the rotational Brownian motion of a dipole in a potential. This procedure sets the various relaxation mechanisms rigorously in the context of the general theory of stochastic processes. Moreover, it allows one to consider a continuous distribution of orien- tations and the inclusion of the intrawell as well as the overbarrier processes in the overall relaxation. In addition, it becomes apparent that a Stosszahlansatz other than the adiabatic collision mechanism underlying the Brownian motion may also be treated. This fact is of paramount importance in the extension of the theory of normal orientational relaxation in an asymmetric bistable potential to model anomalous orientational relaxation behavior such as occurs in glass-forming liquids [8].

By way of introduction, we remark that adaptations of Einstein's theory of the Brownian motion in the presence of an external potential [9], have been widely used to model exponentially decaying relaxation phenomena. In particular, we mention the Debye model of orientational relaxation [4] constituting an adaptation of Einstein's translational Brownian motion calculations to rotational motion in a potential arising from the combined effect of a mean field due to other molecules and an applied external time varying field. Applications of the Debye model include dielectric relaxation and the dynamic Kerr effect of nematic liquid crystals, magnetic relaxation of single domain ferromagnetic particles, dynamic response of Josephson tunneling junctions, structural relaxation processes in amorphous materials such as glassforming liquids, etc. [6,9]. The Debye model assumes that the dipole reorientation is governed by a single particle distribution function with generator given by a Markov process evolving in time as a sequence of small angular steps caused by collisions with the surrounding molecules as well as under the effect of torques originating from, in the most general case, an asymmetric internal mean field potential and an applied external field. Moreover, the Debye theory (like Einstein's theory) is based on the Fokker-Planck equation arising from the continuum limit of a discrete time random walk in configuration space [6]. Thus a characteristic microscopic 
time scale is involved, namely, the mean square distance that a Brownian particle may travel in a small time $\Delta t$. The existence of such a microscopic time scale taking Einstein's original calculation of the mean square displacement of a Brownian particle as an example, always leads as a consequence of the central limit theorem [6] to Gaussian behavior of the distribution function. If such a microscopic time scale does not exist (e.g., where the Brownian particle is trapped in a particular configuration for an arbitrarily long time before making a jump so that we have a chaotic distribution of waiting times as will arise in disordered materials), the central limit theorem no longer applies leading to anomalous diffusion and so to anomalous relaxation behavior. The fundamental solution or Green's function in this case is no longer Gaussian, instead [10] it is a Lévy distribution with its characteristic long time tails giving rise to anomalous diffusion and relaxation.

Here referring to the normal diffusion, the Fokker-Planck equation is a partial differential equation for the evolution of the distribution function of orientations of the molecule in configuration space and so applies to strong dissipative coupling to the heat bath [11]. Thus the Debye theory always assumes that the dipolar molecule is bound so strongly to the surrounding molecules (bath) that large jumps of the dipole direction are extremely unlikely $[3,11]$. This according to Fröhlich [3] may be true in a number of cases but others may exist in which the opposite (large jumps) is much more likely. A dipolar molecule will then make many jumps due to thermal agitation over the potential barriers separating it from another dipole direction during the time required for an appreciable change in direction by viscous flow. Clearly this holds for solids where flow may be considered as entirely absent; however it may also be expected in liquids (such as liquid crystals) where the viscosity is so high that flow is practically negligible. Moreover, both large and small jump transitions may exist simultaneously giving rise to occasional large angle reorientations (with exponentially large relaxation times) of the dipole over many potential barriers. Thus we have both solid and liquidlike behavior in the bistable potential model. The large jumps and the longest lived (overbarrier) relaxation mode (an Arrhenius-like process) which is associated with them may essentially be described by the Kramers escape rate for a heavily damped Brownian particle since the noninertial limit is assumed. There the energy loss per cycle of a particle with energy equal to the saddle point energy of the potential is much greater than the thermal energy $k T$. Thus in normal diffusion the relaxation time associated with the overbarrier process is exponentially long $[6,7]$. As well as the overbarrier process, fast intrawell relaxation processes exist having near degenerate eigenvalues and so may be characterized by a single fast relaxation mode. One may then show by solving the Fokker-Planck equation using matrix continued fraction methods, how simple and accurate approximate formulas for the longitudinal and transverse components of the complex dielectric susceptibility and the corresponding relaxation times may be obtained. These formulas are based on the exponential separation of the time scales of the intrawell and overbarrier (interwell) relaxation processes. Thus a complete analytical solution of the normal longitudinal dielectric (or magnetic) relaxation in an asymmetric double-well potential is given by two Lorentzians and so is almost as simple in form as the original Debye theory of relaxation in the absence of the potential (see Ref. 6, Sec. 8.3.2 for detail). We coin the term DebyeFröhlich models for such theoretical descriptions of the relaxation behavior.

Despite the success of the Debye theory in qualitatively explaining the dynamical behavior of relaxing systems many disordered substances (in the sense that they lack the periodicity of crystals but behave mechanically like solids [8]) exist such as [12] glass-forming liquids and polymers showing very significant departures from the Debye behavior [4]. Such behavior is characterized by a very broadband low frequency dielectric loss curve called the primary or $\alpha$-relaxation process with extremely long relaxation times [1] extending over several decades of frequency. This phenomenon is accompanied by a high frequency relaxation process known as the $\beta$ process and a dramatic slowing down of the primary relaxation process near the liquid glass transition. The behavior of the shear viscosity $\kappa$ in glass-forming liquids and solids (which effectively determines the frequency of maximum loss in the $\alpha$ process) follows the VogelTammann-Fulcher law [8]

$$
\kappa=A \exp \left[B /\left(T-T_{g}\right)\right],
$$

where $A$ and $B$ are temperature independent constants. The result of this behavior is that near the glass transition temperature $T_{g}$ the frequency of maximum absorption of the $\alpha$ process becomes zero. The theoretical explanation of this primary process is one of the main challenges in the physics of glasses [8]. Here we shall for the most part discuss the accompanying $\beta$ process. This relaxation process was observed experimentally by Johari and Goldstein in a chlorobenzene-decalin glass-forming system [13] and found to be an Arrhenius or overbarrierlike relaxation process at temperatures both above and below $T_{g}$. These observations suggest that the $\beta$ process may be described by the DebyeFröhlich model where the overbarrier relaxation time is exponential in the reduced barrier height and the overbarrier relaxation process is a simple exponential decay. However, recent measurements by a number of groups [1,14-16] have shown that for some supercooled liquids the slope of the (Arrhenius) plot for the frequency of maximum dielectric loss versus the inverse temperature changes at temperatures close to $T_{g}$ especially above $T_{g}$ indicating that the $\beta$ process also slows down so that it is characterized by a nonexponential decay.

The temporal and spectral features associated with all these phenomena cannot be explained using a normal diffusion model. Such models always rely as explained above on the concept of a well-defined microscopic time scale, namely, the time for an elementary jump of the random walker so that a discrete time random walk is always involved leading ultimately to an exponentially decaying relaxation process. The very long relaxation times encountered in the experimental observations discussed above suggest that such behavior could be described via the language of continuous time random walks (CTRW). Such walks are based on the idea that the length of a given jump of a random walker as well as the 
waiting time between two successive jumps are random variables. This idea was introduced by Montroll and Weiss in $1965[17,18]$. The CTRW concept is the starting point of all attempts to extend the Debye theory to anomalous relaxation in complex systems, so that empirical decay functions used to explain the broad band dielectric loss, e.g., the stretched exponential of Williams and Watts [19], may be justified [20-24]. In particular, the Debye theory of dielectric relaxation of an assembly of non-interacting polar molecules is reformulated using a fractional noninertial Fokker-Planck equation for the purpose of extending that theory to explain anomalous dielectric relaxation in the context of Ref. [22] to provide a microscopic model for the Cole-Cole relaxation.

It may be shown [11] that the fractional Fokker-Planck equation arises from the diffusion limit of the long rests or fractal time random walk where the mean waiting time diverges, however the second moment of the jump length distribution remains finite. The fractal time random walk invariably leads to subdiffusion as the random walker always risks being trapped in some site for an arbitrarily long time before it can advance a distance equal to the finite variance of the jump length distribution due to the disordered character of the medium. The microscopic disorder in effect creates a jagged array of microscopic potential barriers as schematically described in Ref. [25] giving rise to the chaotic distribution of waiting times because the time of jump over a microscopic barrier is a fractal time Poisson process. Models of the fractal time random walk based on the idea of a jagged array of microscopic potential barriers are often called random activation energy models. Such a picture of the fractal time process also suggests that the anomalous exponent, characteristic of the Cole-Cole relaxation, should be temperature dependent, which is in accordance with experimental observations. It has been shown by solving the particular case of rotation of an assembly of dipolar particles $[6,22,26]$ that the fractal time random walk model can reproduce nonexponential Cole-Cole-type anomalous dielectric relaxation behavior. Moreover, the model reduces to the classical Debye model of rotational diffusion when the anomalous exponent is unity. However, no calculations were carried out for the anomalous relaxation in space of an assembly of dipoles in an asymmetric bistable potential, which is the case of greatest interest in structural relaxation. A rigorous calculation based on a fractional Fokker-Planck equation will thus allow one to determine the effect of the anomalous behavior, i.e., the fractal time Stosszahlansatz, on all the decay modes of the system particularly the slowing down of the overbarrier mode associated with the Kramers escape rate and to calculate the dynamic dielectric susceptibility.

Here we shall evaluate the components of the dielectric susceptibility tensor of an assembly of noninteracting polar Brownian particles in the asymmetric double-well mean field potential arising from a uniaxial potential $-K(\mathbf{n} \cdot \mathbf{e})^{2}(K$ is the anisotropy constant, $\mathbf{e}=\boldsymbol{\mu} / \boldsymbol{\mu}, \boldsymbol{\mu}$ is the permanent dipole moment of a molecule, and $\mathbf{n}$ is the unit vector in the direction of the anisotropy axis) and a superimposed strong dc electric field $\mathbf{E}_{0}$. In order to retain axial symmetry, we suppose that the field $\mathbf{E}_{0}$ and axis of the uniaxial anisotropy potential $\mathbf{n}$ are directed along the $Z$ axis of the laboratory coordinate system. The total potential can be written as

$$
V_{0}=-K(\mathbf{n} \cdot \mathbf{e})^{2}-\mu\left(\mathbf{E}_{0} \cdot \mathbf{e}\right) .
$$

Both exact and approximate solutions of the fractional Fokker-Planck equation, in which the inertial effects are neglected [26], are presented for this particular bistable asymmetric potential. The exact solution of the problem reduces to the solution of infinite hierarchies of differentialrecurrence relations for the corresponding relaxation functions. The longitudinal and transverse components of the susceptibility tensor may be calculated exactly by matrix continued fractions from the Laplace transform of these relaxation functions using linear response theory. We shall also demonstrate that the characteristic times of the normal diffusion process, namely, the integral and effective relaxation times allow one to evaluate the dielectric response for anomalous diffusion as proposed in Ref. [26]. It will be shown how this procedure yields a simple analytical equation for the complex dielectric susceptibility tensor describing the anomalous relaxation of the system. Finally, we indicate how our results may explain the observed nonArrhenius behavior of the $\beta$ relaxation in glass-forming liquids.

\section{FRACTIONAL ROTATIONAL DIFFUSION EQUATION}

We suppose that a small probing field $\mathbf{E}_{1}$ having been applied to the assembly of dipoles in the distant past $(t=$ $-\infty)$ so that equilibrium conditions have been attained at time $t=0$, is switched off at $t=0$. In addition, it is supposed that the field $\mathbf{E}_{1}$ is weak (i.e., $\mu E_{1} \ll k T$, which is the linear response condition; $k$ is the Boltzmann constant, and $T$ is the temperature). Our starting point is the Fokker-Planck equation for the evolution of the probability density function $W(\vartheta, \varphi, t)$ for normal diffusion of dipole moment orientations on the unit sphere in configuration space $(\vartheta$ and $\varphi$ are the polar and azimuthal angles of the dipole, respectively). This equation is valid when the inertia of a dipole is neglected (meaning very strong dissipative coupling to the bath) and has the form

$$
\frac{\partial}{\partial t} W=L_{\mathrm{FP}} W .
$$

The Fokker-Planck operator $L_{\mathrm{FP}}=L_{\mathrm{FP}}^{0}+L_{\mathrm{ext}}$ for normal rotational diffusion is defined as $[6,9]$

$$
\begin{gathered}
L_{\mathrm{FP}}^{0} W=\frac{1}{2 \tau}\left[\boldsymbol{\nabla}^{2} W+\frac{1}{k T} \boldsymbol{\nabla}\left(W \boldsymbol{\nabla} V_{0}\right)\right], \\
L_{\mathrm{ext}} W=\frac{1}{2 \tau k T} \boldsymbol{\nabla}\left(W \boldsymbol{\nabla} V_{\gamma}\right),
\end{gathered}
$$

where $\boldsymbol{\nabla}^{2}$ and $\boldsymbol{\nabla}$ are the Laplacian and gradient on the surface of the unit sphere, $\tau=\zeta / 2 k T$ is the Debye relaxation time for rotation in space, $\zeta$ is the viscous drag coefficient. The potential energy $V_{\gamma}$ of a dipole due to the perturbing field $\mathbf{E}_{1}$ is (see Fig. 1)

$$
V_{\gamma}(\vartheta, \varphi, t)=-\mu E_{1}(t) u_{\gamma}(\vartheta, \varphi) .
$$

Here $\gamma=\|$ denotes the longitudinal response and $\gamma=\perp$ for the transverse response with $u_{\|}=\cos \vartheta$ and $u_{\perp}=\cos \varphi \sin \vartheta$. 


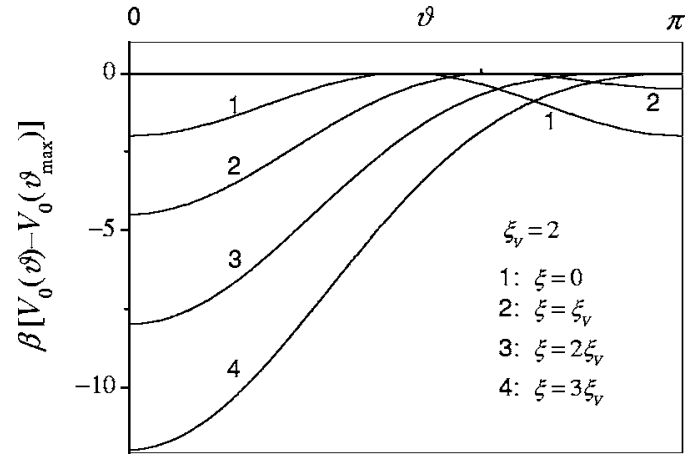

FIG. 1. Double-well potential $V_{0}(\vartheta) / k T=-\xi_{V} \cos ^{2} \vartheta-\xi \cos \vartheta$ for anisotropy (inverse temperature) parameter $\xi_{V}=2$ and various values of the bias field parameter $\xi$ ( $\xi=0$ corresponds to symmetric bistable potential).

Our objective is to ascertain how anomalous diffusion modifies the dielectric relaxation in a bistable potential with nonequivalent wells, Eq. (1) (see Fig. 1). Thus we need the fractional Fokker-Planck equation describing the evolution of the distribution function of orientations of a dipole with the underlying mechanism (or Stosszahlansatz) being a fractal time random walk rather than the fixed jump time random walk underlying the normal diffusion Eq. (1). The fractional Fokker-Planck equation for the diffusion limit of a fractal time random walk on the surface of a unit sphere may be written down by adapting calculations [27-29] given for the diffusion limit of the translational fractal continuous time random walk to the rotational motion of a rigid dipole so that $[6,11]$

$$
\frac{\partial W}{\partial t}=\tau^{1-\sigma}{ }_{0} D_{t}^{1-\sigma} L_{\mathrm{FP}} W
$$

where the operator ${ }_{0} D_{t}^{1-\sigma} \equiv \partial / \partial t_{0} D_{t}^{-\sigma}$ in Eq. (5) is given in terms of the convolution (the Riemann-Liouville fractional integral definition) [27,30]

$$
{ }_{0} D_{t}^{-\sigma} W(\vartheta, \varphi, t)=\frac{1}{\Gamma(\sigma)} \int_{0}^{t} \frac{W\left(\vartheta, \varphi, t^{\prime}\right) d t^{\prime}}{\left(t-t^{\prime}\right)^{1-\sigma}},
$$

where $\Gamma(z)$ is the gamma function [31]. Here, we consider subdiffusion phenomena only $(0<\sigma<1 ; \sigma=1$ corresponds to the normal diffusion).

The formal step-off transient solution of Eq. (5) for $t \geqslant 0$ is obtained from the eigenfunction representation [26,29]

$$
W(\vartheta, \varphi, t)=W_{0}(\vartheta)+\sum_{p=0}^{\infty} \Phi_{p}^{\gamma}(\vartheta, \varphi) F_{p}(t)
$$

with the initial (equilibrium) distribution function

$$
\begin{aligned}
W(\vartheta, \varphi, 0) & =C e^{-\left[V_{0}(\vartheta)+V_{\gamma}(\vartheta, \varphi, 0)\right] / k T} \\
& =W_{0}(\vartheta)\left[1+\xi_{1}\left(u_{\gamma}-\left\langle u_{\gamma}\right\rangle_{0}\right)+O\left(\xi_{1}^{2}\right)\right],
\end{aligned}
$$

where $W_{0}(\vartheta)=e^{-V_{0} / k T} / Z$ is the equilibrium distribution function in the absence of the external field $\mathbf{E}_{1}$, which satisfies $L_{\mathrm{FP}}^{0} W_{0}(\vartheta)=0, Z$ is the partition function given by

$$
\begin{aligned}
Z & =\int_{-1}^{1} e^{\xi x+\xi_{V} x^{2}} d x \\
& =\sqrt{\frac{\pi}{4 \xi_{V}}} e^{-\xi_{V} h^{2}}\left\{\operatorname{erfi}\left[\sqrt{\xi_{V}}(1+h)\right]+\operatorname{erfi}\left[\sqrt{\xi_{V}}(1-h)\right]\right\}
\end{aligned}
$$

$\operatorname{erfi}(x)=2 / \sqrt{\pi} \int_{0}^{x} e^{t^{2}} d t$ is the error function of imaginary argument, $h=\xi /\left(2 \xi_{V}\right)$ is the reduced field (or asymmetry) parameter, $\xi_{V}=K / k T$ and $\xi=\mu E_{0} / k T$ are the dimensionless anisotropy and bias field parameters, $\xi_{1}=\mu E_{1} / k T$ is the applied external field parameter, the angular brackets $\langle\cdots\rangle_{0}$ mean the equilibrium statistical average over $W_{0}$, and the decay modes $F_{p}(t)$ obey the equation

$$
\frac{d}{d t} F_{p}(t)=-\lambda_{p, \sigma 0}^{\gamma} D_{t}^{1-\sigma} F_{p}(t)
$$

Here the eigenvalues $\lambda_{p, \sigma}^{\gamma}$ are expressed in terms of the eigenvalues $\lambda_{p}^{\gamma}$ of the operator $L_{\mathrm{FP}}^{0}$ for the normal diffusion, viz.,

$$
L_{\mathrm{FP}}^{0} \Phi_{p}^{\gamma}(\vartheta, \varphi)=-\lambda_{p}^{\gamma} \Phi_{p}^{\gamma}(\vartheta, \varphi)
$$

so that $[26,29]$

$$
\lambda_{p, \sigma}^{\gamma}=\lambda_{p}^{\gamma} \tau^{1-\sigma}
$$

The solution of Eq. (10) is given by $[27,29]$

$$
F_{p}(t)=E_{\sigma}\left(-\lambda_{p, \sigma}^{\gamma} t^{\sigma}\right)
$$

where $E_{\sigma}\left(-\lambda_{p, \sigma}^{\gamma} t^{\sigma}\right)$ is the Mittag-Leffler function defined as

$$
E_{\sigma}(z)=\sum_{n=0}^{\infty} \frac{z^{n}}{\Gamma(1+\sigma n)} .
$$

The Mittag-Leffler function interpolates between an initial stretched exponential form or Kohlrausch-Williams-Watts function [8]

$$
E_{\sigma}\left[-(t / \tau)^{\sigma}\right] \sim \exp \left[-(t / \tau)^{\sigma} / \Gamma(1+\sigma)\right]
$$

and the long-time inverse power-law behavior characteristic of the long-time behavior of the moments of a Lévy distribution [10]

$$
E_{\sigma}\left(-(t / \tau)^{\sigma}\right) \sim\left[(t / \tau)^{\sigma} \Gamma(1-\sigma)\right]^{-1} t \gg \tau, \quad 0<\sigma<1 .
$$

Equation (11) exemplifies how the eigenvalues of the normal distribution process are altered, in this case reduced, by the nonlocal character of the anomalous diffusion process. The eigenvalues of that process are related to their Brownian counterparts by the prefactor $\tau^{1-\sigma}$.

The Sturm-Liouville representation (7) is purely a formal solution as a knowledge of all eigenfunctions $\Phi_{p}^{\gamma}(\vartheta, \varphi)$ and corresponding eigenvalues $\lambda_{p}^{\gamma}$ is required. However, this representation is very useful as it allows one to obtain a formal solution for the dipole relaxation functions $C_{\gamma}(t)$ and the complex susceptibility tensor components $\chi_{\gamma}(\omega)=\chi_{\gamma}^{\prime}(\omega)$ $-i \chi_{\gamma}^{\prime \prime}(\omega),(\gamma=\|, \perp)$. According to linear response theory [6], the longitudinal and transverse components of the complex dielectric susceptibility $\chi_{\gamma}(\omega)$ are defined as 


$$
\frac{\chi_{\gamma}(\omega)}{\chi_{\gamma}}=1-i \omega \int_{0}^{\infty} e^{-i \omega t} C_{\gamma}(t) d t
$$

where

$$
\begin{gathered}
C_{\gamma}(t)=\frac{\left\langle u_{\gamma}\right\rangle(t)-\left\langle u_{\gamma}\right\rangle_{0}}{\left\langle u_{\gamma}\right\rangle(0)-\left\langle u_{\gamma}\right\rangle_{0}}, \\
\chi_{\|}=\frac{\mu^{2} N_{0}}{k T}\left(\left\langle\cos ^{2} \vartheta\right\rangle_{0}-\langle\cos \vartheta\rangle_{0}^{2}\right)
\end{gathered}
$$

and

$$
\chi_{\perp}=\frac{\mu^{2} N_{0}}{k T}\left\langle\cos ^{2} \varphi \sin ^{2} \vartheta\right\rangle_{0}
$$

are the components of the static susceptibility tensor, $N_{0}$ is the concentration of dipoles, and $\langle\cdots\rangle(t)$ denotes the statistical averages $W(\vartheta, \varphi, t)$. Thus one has from Eqs. (7) and (12)-(14) [26]

$$
C_{\gamma}(t)=\sum_{p} c_{p}^{\gamma} E_{\sigma}\left[-\lambda_{p}^{\gamma} \tau(t / \tau)^{\sigma}\right]
$$

and

$$
\frac{\chi_{\gamma}(\omega)}{\chi_{\gamma}}=\sum_{p} \frac{c_{p}^{\gamma}}{1+(i \omega \tau)^{\sigma} /\left(\tau \lambda_{p}^{\gamma}\right)},
$$

where $\Sigma_{p} c_{p}^{\gamma}=1$

In the low- $(\omega \rightarrow 0)$ and high- $(\omega \rightarrow \infty)$ frequency limits, the susceptibility tensor components may readily be evaluated. We have from Eq. (16), respectively, for $\omega \rightarrow 0$ and for $\omega \rightarrow \infty$

$$
\begin{gathered}
\frac{\chi_{\gamma}(\omega)}{\chi_{\gamma}} \approx 1-\frac{\tau_{\text {int }}^{\gamma}}{\tau}(i \omega \tau)^{\sigma}+\cdots, \\
\frac{\chi_{\gamma}(\omega)}{\chi_{\gamma}} \sim \frac{\tau}{(i \omega \tau)^{\sigma} \tau_{\mathrm{ef}}^{\gamma}}+\cdots,
\end{gathered}
$$

where

$$
\tau_{\text {int }}^{\gamma}=\sum_{p} c_{p}^{\gamma} / \lambda_{p}^{\gamma} \quad \text { and } \quad \tau_{\text {ef }}^{\gamma}=1 / \sum_{p} c_{p}^{\gamma} \lambda_{p}^{\gamma} .
$$

Here $\tau_{\text {int }}^{\gamma}$ is the integral relaxation time (the area under the relaxation function) and $\tau_{\mathrm{ef}}^{\gamma}$ is the effective relaxation time (yielding precise information on the initial decay of the relaxation function in the time domain) for normal diffusion $(\sigma=1)$. Indeed, the relaxation times $\tau_{\text {int }}^{\gamma}$ and $\tau_{\mathrm{ef}}^{\gamma}$ can be evaluated from their equivalent definitions [6]

$$
\tau_{\text {int }}^{\gamma}=\int_{0}^{\infty} C_{\gamma}(t) d t \quad \text { and } \quad \tau_{\mathrm{ef}}^{\gamma}=-1 / \dot{C}_{\gamma}(0) .
$$

On the other hand [6] no such characteristic times exist in anomalous diffusion $(\sigma<1)$ because of the underlying chaotic waiting time distribution which is the generator of the fractal time random walk. This is obvious from the long-time inverse power law behavior of the Mittag-Leffler function. In the context of anomalous diffusion, the times $\tau_{\text {int }}^{\gamma}$ and $\tau_{\text {ef }}^{\gamma}$ are always parameters of the normal diffusion. They exist because in normal diffusion an underlying microscopic time scale exists, namely, the duration of an elementary jump, characteristic of the random walk.

\section{MATRIX CONTINUED FRACTION SOLUTION}

In general, it is difficult to evaluate dielectric parameters from Eqs. (16) as a knowledge of all the eigenvalues $\lambda_{k}^{\gamma}$ and corresponding amplitudes $c_{k}^{\gamma}$ is required. A simpler (from the computational point of view) solution can be given via matrix continued fractions [6]. The general transient response solution of Eq. (5) for $t \geqslant 0$ is [6]

$$
\begin{aligned}
W(\vartheta, \varphi, t)= & W_{0}(\vartheta) \\
& +\frac{1}{4 \pi} \sum_{l=0}^{\infty} \sum_{m=-l}^{l} \frac{(2 l+1)(l-m) !}{(l+m) !} c_{l, m}^{\gamma}(t) e^{i m \varphi} P_{l}^{m}(\cos \vartheta),
\end{aligned}
$$

where the $P_{l}^{m}(x)$ are the associated Legendre functions [31] and the equilibrium distribution function may be expanded in the Legendre polynomials $P_{l}(x)$ [31], viz.,

$$
W_{0}(\vartheta)=\frac{1}{4 \pi} \sum_{l=0}^{\infty}(2 l+1) G_{l} P_{l}(\cos \vartheta),
$$

and $G_{l}=\left\langle P_{l}(\cos \vartheta)\right\rangle_{0}$. By substituting Eq. (21) into Eq. (5), we have the fractional differential recurrence relations for the longitudinal and transverse relaxation functions $f_{l}^{\|}(t)$ and $f_{l}^{\perp}(t)$

$$
\begin{aligned}
\frac{d}{d t} f_{l}^{\|}(t)= & \tau^{-\sigma}{ }_{0} D_{t}^{1-\sigma}\left\{\left[\frac{\xi_{V} l(l+1)}{(2 l-1)(2 l+3)}-\frac{l(l+1)}{2}\right] f_{l}^{\|}(t)\right. \\
& +\frac{\xi}{2(2 l+1)}\left[f_{l-1}^{\|}(t)-f_{l+1}^{\|}(t)\right]+\frac{\xi_{V}(l-1)}{(2 l-1)(2 l+1)} f_{l-2}^{\|}(t) \\
& \left.-\frac{\xi_{V}(l+2)}{(2 l+1)(2 l+3)} f_{l+2}^{\|}(t)\right\},
\end{aligned}
$$

$$
\begin{aligned}
\frac{d}{d t} f_{l}^{\perp}(t)= & \tau_{0}^{-\sigma} D_{t}^{1-\sigma}\left\{\left[\xi_{V} \frac{l(l+1)-3}{(2 l-1)(2 l+3)}-\frac{l(l+1)}{2}\right] f_{l}^{\perp}(t)\right. \\
& +\frac{\xi}{2(2 l+1)}\left[(l+1)^{2} f_{l-1}^{\perp}(t)-l^{2} f_{l+1}^{\perp}(t)\right] \\
& \left.+\frac{\xi_{V} l(l+1)^{2}}{(2 l-1)(2 l+1)} f_{l-2}^{\perp}(t)-\frac{\xi_{V} l^{2}(l+1)}{(2 l+1)(2 l+3)} f_{l+2}^{\perp}(t)\right\},
\end{aligned}
$$

where $f_{l}^{\|}(t)$ and $f_{l}^{\perp}(t)$ are defined by

$$
\begin{gathered}
f_{l}^{\|}(t)=c_{l, 0}^{\|}(t)=\left\langle P_{l}(\cos \vartheta)\right\rangle(t)-\left\langle P_{l}\right\rangle_{0}, \\
f_{l}^{\perp}(t)=\operatorname{Re}\left[c_{l, 1}^{\perp}(t)\right]=\left\langle\cos \varphi P_{l}^{1}(\cos \vartheta)\right\rangle(t),
\end{gathered}
$$

so that the relaxation function $C_{\gamma}(t)$ describing the decay of the electric polarization is $C_{\gamma}(t)=f_{1}^{\gamma}(t) / f_{1}^{\gamma}(0)$.

We now present the solution of Eqs. (23) and (24) and in terms of matrix continued fractions. The advantage of posing 
the problem in this way is that exact formulas in terms of such continued fractions may be written for the Laplace transform of the after-effect function, the correlation time, and the complex susceptibility. The starting point of the calculation is Eqs. (7) and (8) written as the matrix differential recurrence relation

$$
\dot{\mathbf{C}}_{l}^{\gamma}(t)=\tau^{-\sigma}{ }_{0} D_{t}^{1-\sigma}\left[\mathbf{Q}_{l}^{\gamma-} \mathbf{C}_{l-1}^{\gamma}(t)+\mathbf{Q}_{l}^{\gamma} \mathbf{C}_{l}^{\gamma}(t)+\mathbf{Q}_{l}^{\gamma+} \mathbf{C}_{l+1}^{\gamma}(t)\right] .
$$

Here the vectors $\mathbf{C}_{l}^{\gamma}(t)$ are defined as

$$
\mathbf{C}_{l}^{\gamma}(t)=\left(\begin{array}{c}
f_{2 l-1}^{\gamma}(t) \\
f_{2 l}^{\gamma}(t)
\end{array}\right),
$$

where the $2 \times 2$ matrices $\mathbf{Q}_{l}^{\gamma \pm}, \mathbf{Q}_{l}^{\gamma}$ are given in the Appendix. The three-term matrix recurrence relation Eq. (27) may now be solved for the Fourier-Laplace transform $\widetilde{\mathbf{C}}_{1}^{\gamma}(\omega)$ yielding [6]

$$
\begin{aligned}
\widetilde{\mathbf{C}}_{1}^{\gamma}(\omega)= & \tau(i \omega \tau)^{\sigma-1} \boldsymbol{\Delta}_{1}^{\gamma}(\omega)\left[\mathbf{C}_{1}^{\gamma}(0)\right. \\
& \left.+\sum_{l=2}^{\infty}\left(\prod_{k=2}^{l} \mathbf{Q}_{k-1}^{\gamma+} \boldsymbol{\Delta}_{k}^{\gamma}(\omega)\right) \mathbf{C}_{l}^{\gamma}(0)\right],
\end{aligned}
$$

where $\Delta_{k}^{\gamma}(\omega)$ is the $2 \times 2$ matrix continued fraction defined as

$$
\boldsymbol{\Delta}_{k}^{\gamma}(\omega)=\left[(i \omega \tau)^{\sigma} \mathbf{I}-\mathbf{Q}_{k}^{\gamma}-\mathbf{Q}_{k}^{\gamma+} \boldsymbol{\Delta}_{k+1}^{\gamma}(\omega) \mathbf{Q}_{k+1}^{\gamma-}\right] .
$$

The initial value vectors

$$
\mathbf{C}_{l}^{\gamma}(0)=\left(\begin{array}{c}
f_{2 l-1}^{\gamma}(0) \\
f_{2 l}^{\gamma}(0)
\end{array}\right)
$$

can be also evaluated in terms of such continued fractions (see the Appendix). Here $\widetilde{\mathbf{C}}_{l}^{\gamma}(\omega)$ denotes the one-sided Fourier transform, viz.,

$$
\widetilde{\mathbf{C}}_{l}^{\gamma}(\omega)=\int_{0}^{\infty} e^{-i \omega t} \mathbf{C}_{l}^{\gamma}(t) d t
$$

Equations (29) and (30) and constitute the exact solution of our problem formulated in terms of matrix continued fractions. Having determined the Laplace transform $\tilde{f}_{l}^{\gamma}(\omega)$ and noting that $\widetilde{C}_{\gamma}(i \omega)=\tilde{f}_{1}^{\gamma}(i \omega) / f_{1}^{\gamma}(0)$, one may calculate the susceptibility $\chi_{\gamma}(\omega)$ from Eq. (13).

\section{BIMODAL APPROXIMATION}

The matrix continued fraction method given yields the exact solutions for the complex susceptibility for all values of the thermal and anisotropy energies. Consequently, that method is an indispensable tool in estimating the accuracy of approximate solutions for typical parameters of the system. For example, in normal diffusion, the Kramers escape rate provides a close approximation to the smallest nonvanishing eigenvalue the inverse of which in turn provides an approximation to the longest relaxation time in a system where bar- rier crossing is involved as well as the correlation time for bistable potentials with equivalent wells. The matrix continued fraction method then allows one to determine the range of system parameters, e.g., barrier height, friction, etc., in which the approximate solution for the longest relaxation time provided by the Kramers method is valid. Nevertheless, in practical applications such as the analysis of experimental results, the matrix continued fraction method is of very limited use since the dependence of the susceptibility tensor on the model parameters is not obvious from this method. Thus it is desirable to try to obtain simple approximate formulas describing the dynamical behavior. This has been accomplished in $[6,26]$ for both normal and anomalous diffusion. The method proceeds from linear response theory which allows one to use, taking anomalous diffusion as an example, the after effect solution of the fractional Fokker-Planck Eq. (5) Thus the solution of Eq. (5) may be converted to a SturmLiouville problem with the time behavior described by an infinite set of Mittag-Leffler [6] functions rather than the decaying exponentials characteristic of the normal diffusion. The Mittag-Leffler functions all have a long-time tail characteristic of anomalous diffusion. Moreover, their arguments depend on the eigenvalues of the normal diffusion process. The presence of the long-time tail means that the MittagLeffler functions describe the nonlocal behavior of the system. The advantage of posing the solution of Eq. (5) as a Sturm-Liouville problem now becomes apparent. Namely, by formulating the problem in this way it is very easy to determine how the eigenvalues of the normal diffusion process are scaled (in the case of subdiffusion $\sigma<1$ considered here reduced) by the nonlocal character resulting from the microscopic disorder of the anomalous diffusion process. An important consequence of this is that one may determine the slowing down of the Kramers escape rate in essence the lowering due to nonlocal effects of the frequency of maximum loss (and consequent slowing down of the relaxation process) of the low frequency absorption. (Recall that the Kramers escape rate is the high barrier limit of the smallest nonvanishing eigenvalue of the Fokker-Planck equation for the normal diffusion.) In addition, one may determine how nonlocal effects influence all the other characteristic frequencies of the normal diffusion spectra. Now in the normal longitudinal relaxation, the time scales of the overbarrier and intrawell relaxation processes are exponentially separated. Furthermore, the intrawell modes are near degenerate yielding only two effective relaxation modes. The foregoing considerations suggest that in anomalous longitudinal relaxation one may again approximate the infinite number of MittagLeffler decays, Eq. (15), by two Mittag-Leffler functions only [26]. One of these associated with the overbarrier mode; the other associated with the near degenerate "intrawell" modes.

The corresponding longitudinal complex susceptibility $\chi_{\|}(\omega)$ can be effectively described then by the sum of two Cole-Cole spectra, viz., [26]

$$
\frac{\chi_{\|}(\omega)}{\chi_{\|}}=\frac{\Delta_{1}}{1+\left(i \omega / \omega_{\|}\right)^{\sigma}}+\frac{1-\Delta_{1}}{1+\left(i \omega / \omega_{W}\right)^{\sigma}},
$$

where the characteristic frequencies $\omega_{\|}$and $\omega_{W}$ are given by 


$$
\omega_{\|}=\tau^{-1}\left(\tau \lambda_{1}^{\|}\right)^{1 / \sigma}, \quad \omega_{W}=\tau^{-1}\left(\tau / \tau_{W}\right)^{1 / \sigma}
$$

and $\lambda_{1}^{\|}$is the smallest nonvanishing eigenvalue of the Fokker-Planck operator $L_{\mathrm{FP}}^{0}$ for the normal rotational diffusion defined by Eq. (3). The low-frequency band is due to the slowest (overbarrier) relaxation mode; the characteristic frequency $\omega_{\|}$and the half-width of this band are determined by $\lambda_{1}^{\|}$. Thus, the anomalous low frequency behavior is dominated by the barrier crossing mode as in the normal diffusion. The high-frequency band is due to "intrawell" modes corresponding to the eigenvalues $\lambda_{k}(k \neq 1)$ of $L_{\mathrm{FP}}^{0}$. These near degenerate "intrawell" modes are indistinguishable in the frequency spectrum of $\chi^{\prime \prime}(\omega)$ merely appearing as a single high-frequency band with characteristic frequency $\omega_{W}$. The parameters $\Delta_{1}$ and $\tau_{W}$ are defined in terms of the characteristic times of the normal diffusion (the integral relaxation time $\tau_{\text {int }}^{\|}$, the effective relaxation time $\tau_{\text {ef }}^{\|}$, and the inverse of the smallest non-vanishing eigenvalue $\left.1 / \lambda_{1}^{\|}\right)[6,26]$

$$
\Delta_{1}=\frac{\tau_{\text {int }}^{\|} / \tau_{\text {ef }}^{\|}-1}{\lambda_{1}^{\|} \tau_{\text {int }}^{\|}-2+1 /\left(\lambda_{1}^{\|} \tau_{\text {ef }}^{\|}\right)}, \quad \tau_{W}=\frac{\lambda_{1}^{\|} \tau_{\text {int }}^{\|}-1}{\lambda_{1}^{\|}-1 / \tau_{\text {ef }}^{\|}} .
$$

Furthermore, for the transverse response, noting that $\tau_{\text {int }}^{\perp}$ $\cong \tau_{\text {ef }}^{\perp} \cong \lambda_{1}^{\perp}$ for all values of the model parameters, the spectrum of $\chi_{\perp}(\omega)$ may be approximated by the single Cole-Cole equation

$$
\frac{\chi_{\perp}(\omega)}{\chi_{\perp}}=\frac{1}{1+\left(i \omega / \omega_{\perp}\right)^{\sigma}},
$$

where the characteristic frequency $\omega_{\perp}$ is given by

$$
\omega_{\perp}=\tau^{-1}\left(\tau / \tau_{\mathrm{ef}}^{\perp}\right)^{1 / \sigma} .
$$

In the foregoing equations $\tau_{\text {int }}^{\gamma}, \tau_{\mathrm{ef}}^{\gamma}$, and $\lambda_{1}^{\gamma}$ are calculated as follows. The effective relaxation times $\tau_{\mathrm{ef}}^{\|}$and $\tau_{\mathrm{ef}}^{\gamma}$ are given in terms of equilibrium averages $\left\langle P_{1}\right\rangle_{0}$, and $\left\langle P_{2}\right\rangle_{0}$ only (Ref. [6], Chap. 8)

$$
\begin{gathered}
\tau_{\mathrm{ef}}^{\|}=\tau \frac{2\left\langle P_{2}\right\rangle_{0}+1-3\left\langle P_{1}\right\rangle_{0}^{2}}{1-\left\langle P_{2}\right\rangle_{0}}, \\
\tau_{\mathrm{ef}}^{\perp}=\tau \frac{1-\left\langle P_{2}\right\rangle_{0}}{1+\left\langle P_{2}\right\rangle_{0} / 2},
\end{gathered}
$$

where

$$
\begin{gathered}
\left\langle P_{1}\right\rangle_{0}=\frac{e^{\xi_{V}} \sinh \xi}{\xi_{V} Z}-\frac{\xi}{2 \xi_{V}}, \\
\left\langle P_{2}\right\rangle_{0}=\frac{3 e^{\xi_{V}}}{2 \xi_{V} Z}\left(\cosh \xi-\frac{\xi}{2 \xi_{V}} \sinh \xi\right)+\frac{3 \xi^{2}}{8 \xi_{V}^{2}}-\frac{3}{4 \xi_{V}}-\frac{1}{2},
\end{gathered}
$$

the partition function $Z$ is given by Eq. (9). The integral relaxation time $\tau_{\text {int }}^{\gamma}$ can be evaluated numerically from the continued fraction solution $\tau_{\text {int }}^{\gamma}=\widetilde{C}_{\gamma}(0) / C_{\gamma}(0)$ at $\sigma=1$. Moreover, the longitudinal integral relaxation time $\tau_{\text {int }}^{\|}$is given in exact integral form as ([6], Chap. 8)

$$
\begin{aligned}
\tau_{\text {int }}^{\|}= & \frac{2 \tau}{Z\left(\left\langle\cos ^{2} \vartheta\right\rangle_{0}-\langle\cos \vartheta\rangle_{0}^{2}\right)} \\
& \times \int_{-1}^{1}\left[\int_{-1}^{z}\left(z^{\prime}-\langle\cos \vartheta\rangle_{0}\right) e^{-V_{0}\left(z^{\prime}\right) / k T} d z^{\prime}\right]^{2} \frac{e^{V_{0}(z) / k T}}{1-z^{2}} d z,
\end{aligned}
$$

where $z=\cos \vartheta$. The smallest eigenvalue $\lambda_{1}^{\|}$can also be evaluated numerically as described in detail in Ref. [6], Chap. 8.

In the low temperature limit $2 \xi_{V}+\xi \gg 1$, the quantities $\Delta_{1}$, $1-\boldsymbol{\Delta}_{1}$, and $\tau_{W}$ in Eq. (32) may be evaluated from the simple equations [32]

$$
\begin{gathered}
\chi_{\|} \Delta_{1} \sim \frac{4\left(1-h^{2}\right)}{\left[(1+h) e^{-2 \xi_{V} h}+(1-h) e^{2 \xi_{V} h}\right]^{2}}, \\
\chi_{\|}\left(1-\Delta_{1}\right) \sim\left[2 \xi_{V}(1+h)\right]^{-2}, \\
\tau_{W} \sim \tau\left[2 \xi_{V}(1+h)-\frac{5+h}{1+h}\right]^{-1} .
\end{gathered}
$$

$\lambda_{1}^{\|}$can be approximated by Brown's formula $[33,34]$

$$
\lambda_{1}^{\|}=\frac{\xi_{V}^{3 / 2}\left(1-h^{2}\right)}{\tau \pi^{1 / 2}}\left[(1+h) e^{-\xi_{V}(1+h)^{2}}+(1-h) e^{-\xi_{V}(1-h)^{2}}\right] .
$$

For small values of $\xi_{V}$, Eq. (43) (which assumes high potential barriers) is inadequate. Here, we can use an approximate equation for the effective relaxation time $\tau_{\text {ef }}^{\|}$because $\lambda_{1}^{\|}$ $\approx 1 / \tau_{\text {ef }}^{\|}$for $\xi_{V} \leqslant 1$ [6]. For small values of $\xi$ and $\xi_{V}$, one can use the exact Taylor series expansion ([6], Sec. 8.3.2)

$$
\begin{aligned}
\lambda_{1}^{\|} \tau= & 1-\frac{2}{5} \xi_{V}+\frac{48}{875} \xi_{V}^{2}-\frac{32}{21875} \xi_{V}^{3}+\frac{15552}{58953125} \xi_{V}^{4} \\
& +\left(\frac{1}{10}+\frac{1}{875} \xi_{V}-\frac{686}{84375} \xi_{V}^{2}\right) \xi^{2}-\frac{1}{7000} \xi^{4}+\cdots
\end{aligned}
$$

Selected numerical values of $\tau_{\text {int }}^{\|}, \tau_{\text {ef }}^{\|}, 1 / \lambda_{1}^{\|}, \tau_{W}$, and $\tau_{\text {ef }}^{\perp}$ are presented in Table I. Equations (42) and (43) allow one to readily estimate the asymptotic behavior of the characteristic frequencies $\omega_{\|}$and $\omega_{W}$ in Eq. (33).

Returning to Eqs. (32) and (33) and as far as a physical interpretation is concerned we remark that the peak frequency $\omega_{\|}$of the dielectric loss associated with the overbarrier processes decreases sharply as the anomalous exponent $\sigma$ decreases from unity. Conversely, the overbarrier relaxation time, defined as the inverse peak frequency, sharply increases tending to infinity as $\sigma$ approaches 0 . Moreover, $\sigma$ is in principle temperature dependent as it appears that the microscopic origin of $\sigma$ lies in the random activation energy model which is the ultimate generator of the fractal time random walk underlying our generalized Debye-Fröhlich model. The reason for this behavior is obvious from Eq. (33) because the argument in the exponential of the Arrheniuslike expression for the overbarrier frequency is reduced by the factor $\sigma^{-1}$ due to the scaling of the eigenvalues of the 
TABLE I. Numerical values of $1 / \lambda_{1}^{\|}, \tau_{\text {int }}^{\|}, \tau_{\text {ef }}^{\|}, \tau_{W}$, and $\tau_{\text {ef }}^{\perp}$ for normal diffusion.

\begin{tabular}{ccccc}
\hline \hline$\xi_{V}=10$ & $\xi=0$ & $\xi=2$ & $\xi=5$ & $\xi=10$ \\
\hline $1 / \lambda_{1}^{\|}$ & 693.9 & 232.5 & 26.83 & 2.265 \\
$\tau_{\text {int }}^{\|}$ & 691.0 & 224.15 & 3.398 & 0.0387 \\
$\tau_{\text {ef }}^{\|}$ & 16.64 & 1.690 & 0.0565 & 0.0384 \\
$\tau_{W}$ & 0.0714 & 0.0615 & 0.0495 & 0.0384 \\
$\tau_{\text {ef }}^{\perp}$ & 0.0742 & 0.0661 & 0.0565 & 0.0460 \\
$\xi=2$ & $\xi_{V}=1$ & $\xi_{V}=5$ & $\xi_{V}=10$ & $\xi_{V}=15$ \\
$1 / \lambda_{1}^{\|}$ & 0.9763 & 6.387 & 232.5 & 16484 \\
$\tau_{\text {int }}^{\|}$ & 0.8912 & 5.720 & 224.15 & 16221 \\
$\tau_{\text {ef }}^{\|}$ & 0.7528 & 1.098 & 1.690 & 2.366 \\
$\tau_{W}$ & 0.2866 & 0.1383 & 0.0615 & 0.0377 \\
$\tau_{\text {ef }}^{\perp}$ & 0.3430 & 0.1323 & 0.0661 & 0.0441 \\
\hline \hline
\end{tabular}

normal diffusion caused by the anomalous exponent. Consequently, the overbarrier relaxation mode is extremely sensitive to the value of $\sigma$ and by extension (postulating temperature dependence of $\sigma$ ) to temperature.

\section{RESULTS AND DISCUSSION}

The frequencies $\omega_{\|}$and $\omega_{W}$ as functions of $\xi_{V}$ (i.e., the inverse dimensionless temperature) at a fixed value of $h$ $=0.2$ are plotted in Figs. 2(a) and 3 for various values of $\sigma$ and in Fig. 2(b) for various values of $h$ when $\sigma=0.8$. Apparently the fractional exponent $\sigma$ strongly influences the temperature dependence (expressed by means of the parameter $\left.\xi_{V}\right)$ of $\omega_{\|}$and $\omega_{W}$. The relaxation behavior due to the near degenerate intrawell modes may be (just as the behavior of the overbarrier mode) described by a plot of $\tau \omega_{W}$ as a function of $\xi_{V}$ (Fig. 3). Figure 3 exhibits an increase in the frequency of maximum loss of the high frequency peak due to the intrawell modes as the temperature is reduced. Now a high frequency shoulder appears in the dielectric spectra of a few glass-forming liquids such as glycerol [12] and may be due to the intrawell modes. However, this cannot be rigorously established at present as the temperature dependence of the shoulder frequency is difficult to determine because the amplitude of the shoulder itself is barely detectable in the present state of the art of dielectric spectral measurements. It is also possible that the intrawell mode shoulder may correspond to the mode predicted by Götze and Sjögren in their mode coupling theory of viscous and glass-forming liquids [35].

One of the most interesting facets of the theory is the manner in which the eigenvalues of the normal diffusion process are effectively reduced by the nonlocal character of the anomalous subdiffusion process. In particular, the Kramers escape rate for the present problem is reduced by the factor $\tau^{1-\sigma}$ due to the nonlocal character of the anomalous diffusion. This amounts to a critical slowing down of the overbarrier relaxation mode with consequent broadening of the spectra as $\sigma$ decreases. Moreover, the slowest decay mode will be highly nonexponential in character as its be-
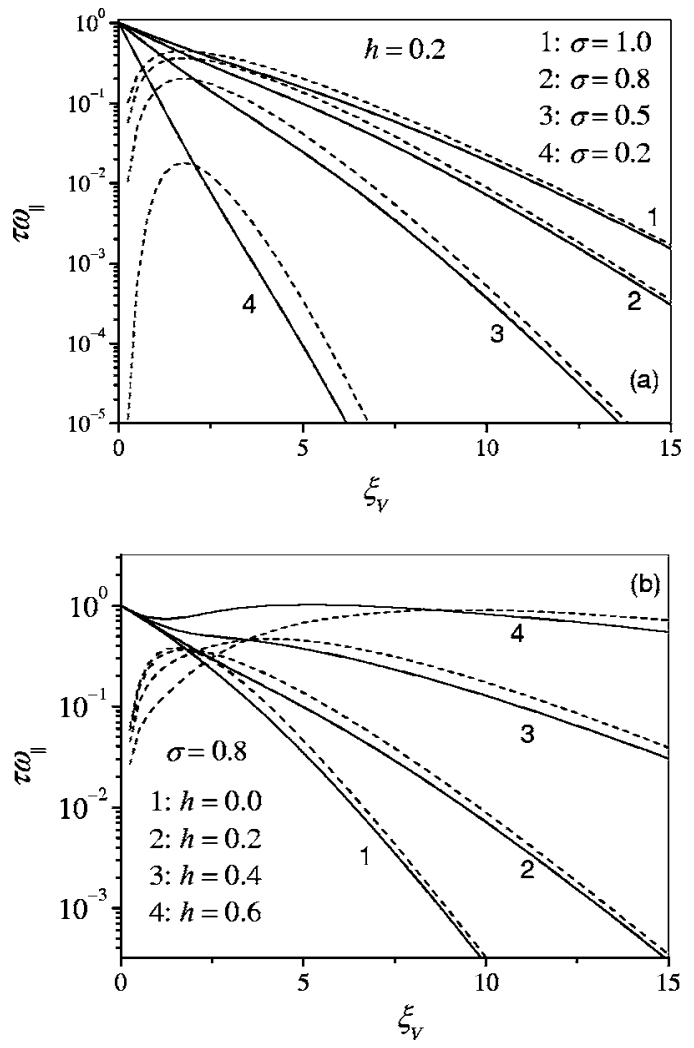

FIG. 2. Angular frequency of maximum loss of Arrhenius process $\omega_{\|}$[solid lines: Eq. (33) with exact values of the smallest nonvanishing eigenvalue $\lambda_{1}^{\|}$] versus $\xi_{V}$ (inverse temperature) for (a) asymmetry parameter $h=0.2$ and various values of anomalous exponent $\sigma$ and for (b) $\sigma=0.8$ and various values of $h$. Dashed lines: Eq. (33) with $\lambda_{1}^{\|}$from the high barrier asymptote Eq. (43).

havior will be dictated by a Mittag-Leffler function with $\omega_{\gamma}$ as part of its argument.

The real and imaginary parts of the susceptibility from both the exact continued fraction solutions and the approximate Eqs. (32) and (35) for typical values of the model parameters are shown in Figs. 4-7. The agreement between the exact continued fraction calculations and the approximate

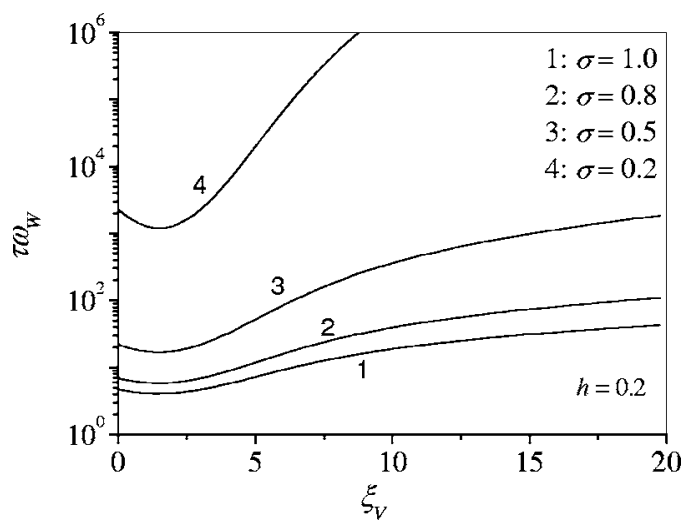

FIG. 3. Well angular frequency of maximum loss $\omega_{W}$ versus. $\xi_{V}$ for $h=0.2$ and various values of $\sigma$ [solid lines: Eqs. (33) and (34) with exact values of the smallest nonvanishing eigenvalue $\lambda_{1}^{\|}$, integral relaxation time $\tau_{\text {int }}^{\|}$, and effective relaxation time $\tau_{\text {ef }}^{\|}$. 

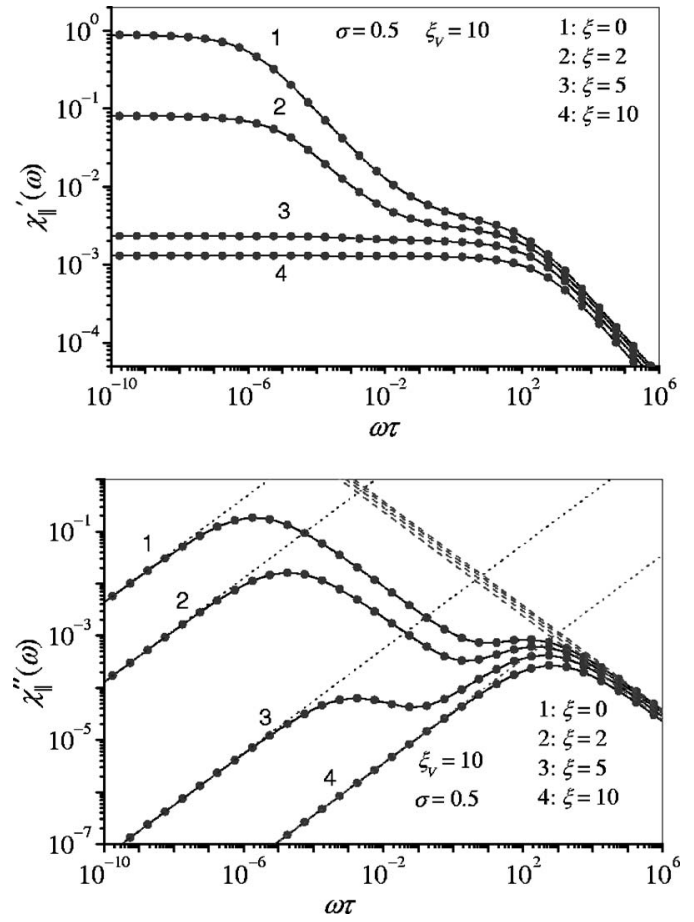

FIG. 4. Real and imaginary parts of the longitudinal complex susceptibility $\chi_{\|}^{\prime}(\omega)$ and $\chi_{\|}^{\prime \prime}(\omega)$ versus $\omega \tau$ evaluated from the exact matrix continued fraction solution (solid lines) for $\sigma=0.5, \xi_{V}=10$ and various values of $\xi$ and compared with those calculated from the approximate Eq. (35) (filled circles) and with the low (dotted lines) and high (dashed lines) frequency asymptotes Eqs. (17) and (18), respectively.
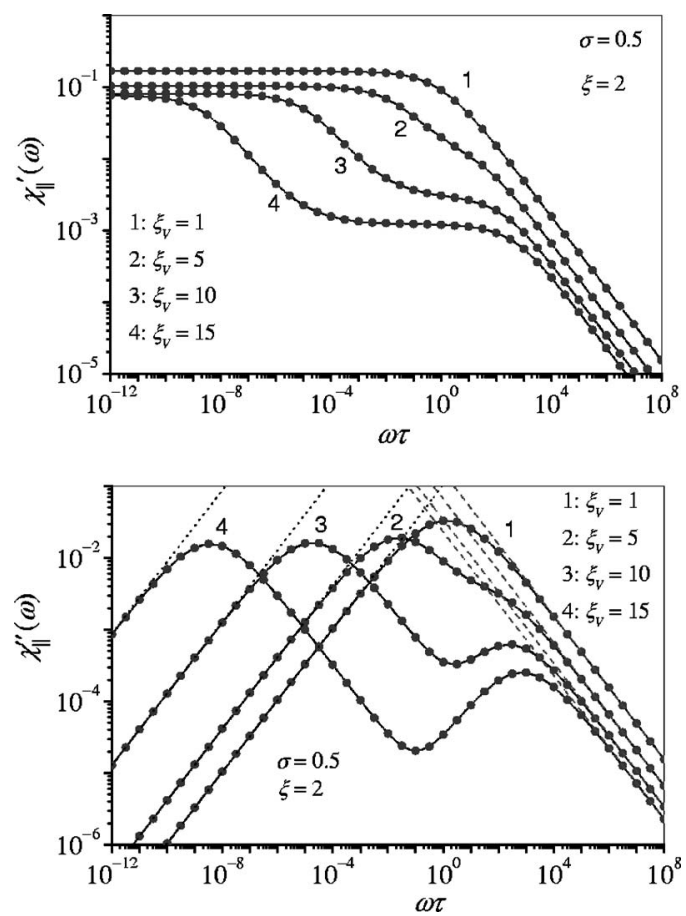

FIG. 5. The same as in Fig. 4 for $\sigma=0.5, \sigma=2$ and various values of $\xi_{V}$.
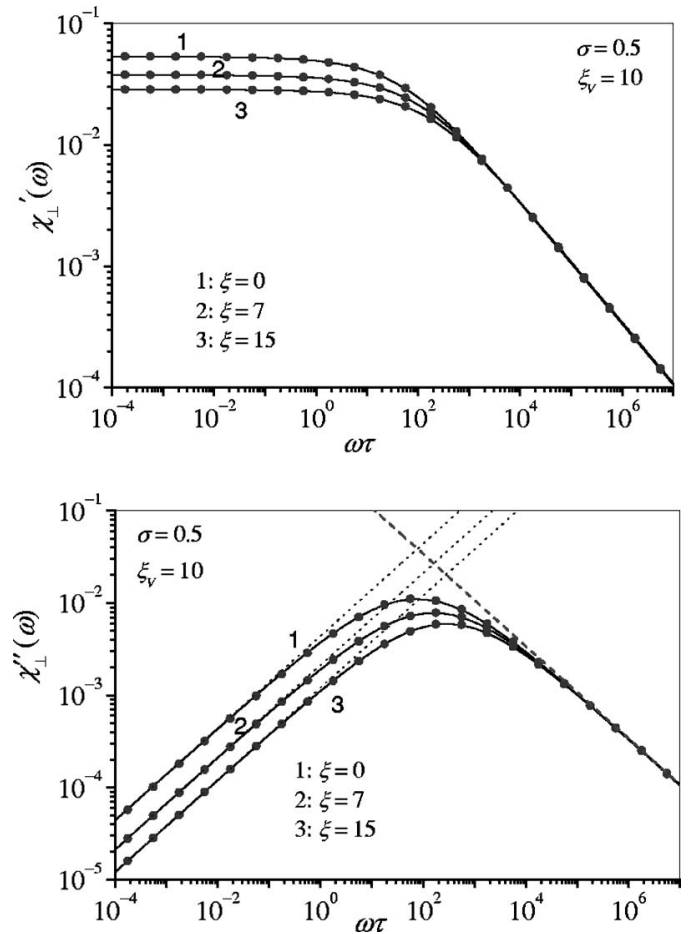

FIG. 6. Real and imaginary parts of the transverse complex susceptibility $\chi_{\perp}^{\prime}(\omega)$ and $\chi_{\perp}^{\prime \prime}(\omega)$ versus $\omega \tau$ evaluated from the exact matrix continued fraction solution (solid lines) for $\sigma=0.5, \xi_{V}=10$, and various values of $\xi$ and compared with those calculated from the approximate Eq. (35) (filled circles) and with the low (dotted lines) and high (dashed lines) frequency asymptotes Eqs. (17) and (18), respectively.

Eqs. (32) and (35) and is very good [the maximum relative deviation between the corresponding curves does not exceed a few (3-5) percent]. Similar (or even better) agreement is obtained for all values of $\xi, \xi_{V}$, and $\sigma$. Thus one may conclude that the Cole-Cole susceptibilities Eqs. (32) and (35) and accurately describe the behavior of $\chi_{\gamma}(\omega)$ for all frequencies of interest and for all values of the bias field strength $(\xi)$, anisotropy constant $\left(\xi_{V}\right)$, and anomalous exponent $(\sigma)$ parameters. Hence, the generalized Debye model can explain the anomalous relaxation of complex dipolar systems where the anomalous exponent $\sigma$ differs from unity (corresponding to the classical Debye theory of dielectric relaxation), i.e., the relaxation process is characterized by a broad distribution of relaxation times. The longitudinal component of the susceptibility tensor in general exhibits a very broad band low frequency peak arising from the overbarrier relaxation superimposed on which is a weaker high frequency wing. However, if the reduced field parameter $h$ (which characterizes the asymmetry of the potential) is sufficiently large $(h>0.5)$, the low frequency peak vanishes which may be explained as follows. We recall that $h=0$ corresponds to the symmetric bistable potential while $h=1$ is the value of $h$ at which the bistable structure of the potential disappears that is the nucleation field. However, for normal diffusion in the asymmetric bistable potential given by Eq. (1) the shallower of the two potential wells becomes completely depopulated at $h \approx 0.17$ which is much less than the 

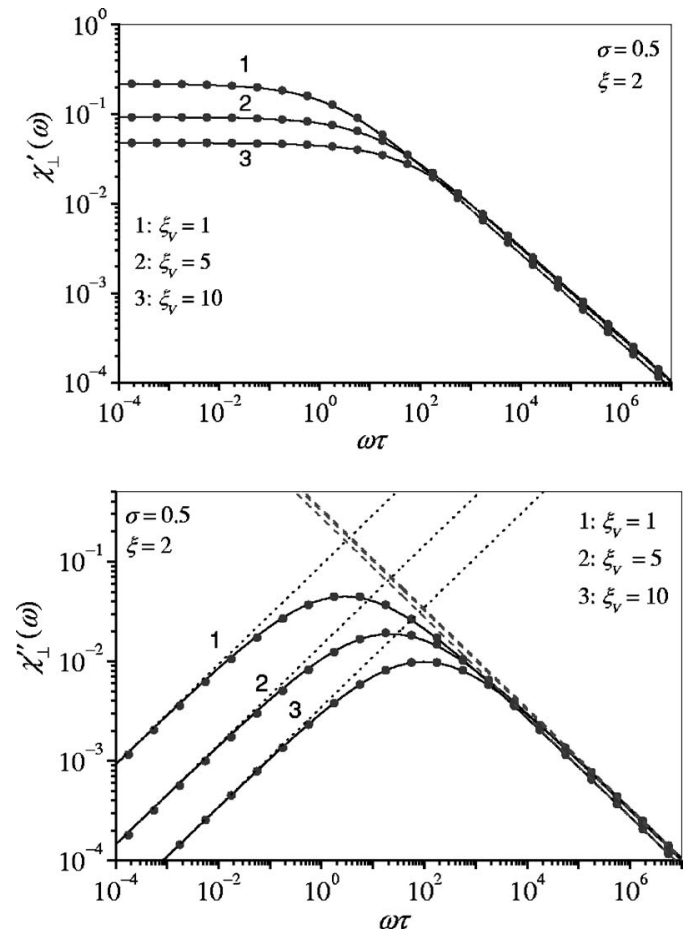

FIG. 7. The same as in Fig. 6 for $\sigma=0.5, \sigma=2$ and various values of $\xi_{V}$.

nucleation field so that the relaxation is no longer dominated by the low frequency peak generated by the overbarrier process. Thus as far as relaxation is concerned although the bistable structure still exists, all that effectively remains is the high frequency absorption peak due to the well process. The behavior is reminiscent of a phase transition controlled by the asymmetry parameter $h$.

As far as comparison with experiment is concerned the theory of the three-dimensional fractional rotational diffusion of rigid dipoles in an asymmetric double-well potential developed in this paper can be applied in qualitative fashion to explain the Johari-Goldstein relaxation $(\beta)$ process in glassforming liquids [13]. This process first appears distinctly at temperatures of about $20 \mathrm{~K}$ above $T_{g}$, and persists at lower temperatures to $30-50 \mathrm{~K}$ below $T_{g}$ in most glass-forming liquids. The process is believed to arise from overbarrier relaxation in the liquid and glass states of matter of dipolar molecules over potential barriers high compared to the thermal energy [36]. We recall that the $\beta$ relaxation process was observed experimentally by Johari and Goldstein in a chlorobenzene-decalin glass-forming system [13] and exhibits Arrhenius-like behavior for the frequency of maximum loss versus $T^{-1}$ at temperatures both above and below $T_{g}$. However, as we have already mentioned recent measurements [14-17] have shown that in a variety of supercooled liquids a significant departure from Arrhenius-like behavior occurs in certain temperature regions, namely, the slope of the Arrhenius plot for the frequency of maximum dielectric loss $\left(f_{m, \beta}\right)$ changes at temperatures close to $T_{g}$ especially above $T_{g}$. An increase in the magnitude of the slope above $T_{g}$ has conclusively been observed for $D$-sorbitol [14] by applying pressure; for picoline-tristyrene mixtures [15], and for the copolyesters of poly(ethylene-terephthalate) and poly(ethylene isophthalate) [16]. In some cases, $f_{m, \beta}$ becomes temperature independent over a small temperature range around $T_{g}$ or even goes through a maximum [1]. We note from Fig. 2 that the plot of $\omega_{\|} \sim f_{m, \beta}$ versus $\xi_{V}$ is observed to be non-Arrhenius in character for values of $\xi_{V}$ in the range $3-6$, where the departure from a straight line is indicated by a kink in the plot. The plot also exhibits the maximum mentioned above [see Fig. 2(b)]. The overall behavior can be understood physically in terms of the asymmetry of the potential landscape altering due to the glass-forming liquid changing its microscopic structure as the temperature of the liquid approaches $T_{g}$ from higher temperatures. After the asymmetry has become well established, the liquid at temperature close to $T_{g}$ is too viscous for any further changes to occur in its energyscape. Thus the plot at lower temperatures $\left(\xi_{V} \gg 1\right)$ again becomes Arrhenius as is experimentally observed. Now, the anomalous exponent $\sigma$ or Cole-Cole parameter is known to vary with temperature [37] as is consistent with the elementary jumps over a jagged potential barrier concept underlying the microscopic origin of the fractal time random walk. Therefore, the plot of $f_{m, \beta}$ versus $1 / T$ may alter its slope as temperature varies indicating a nonArrhenius temperature dependence. This behavior is entirely in accord with that predicted by Eq. (33), where the decrease of $\omega_{\|}$with a decrease in $\sigma$ at a given temperature (specified by $\xi_{V}$ ) is much faster than that predicted by normal diffusion. Thus the effect of anomalous diffusion at a given temperature is to substantially increase the effective barrier height, which becomes infinite as $\sigma \rightarrow 0$. Hence, the overall behavior of $\omega_{\|}$versus $\xi_{V}$ appears to be entirely consistent with the schematic Fig. 1 of Paluch et al. [14] illustrating the relationship between the relaxation times for structural relaxation $(\alpha$ process) and the secondary relaxation or $\beta$ process noting of course that they plot the inverse of $\omega_{\|}$versus inverse temperature.

The theory we have developed may also be applied to dilute suspensions of fine magnetic particles (ferrofluids) by a simple change of notation. Experiments on the magnetization induced by a weak ac field superimposed on a strong dc magnetic field may be realized in practice in a ferrofluid as a large value of $\xi$ can be achieved with a moderate constant magnetic field due to the large value of the magnetic dipole moment $m\left(10^{4}-10^{5}\right.$ Bohr magnetons) of single domain particles. The anomalous relaxation behavior naturally appears in ferrofluids due to the broad distribution of particle volumes $v$ (for fine particles, the magnetic moment and the Debye relaxation time strongly depend on $v$ ) [38]. Again with a simple change of notation the present theory may be used to set the Gilroy and Philips model [2] of structural relaxation processes in amorphous materials and the Dyre and Olsen' minimal model for beta relaxation in viscous liquids [1], which we have essentially discussed in the previous paragraphs, in the framework of the general theory of stochastic processes. Moreover, the formulation of the theory in terms of kinetic equations, as the diffusion limit of fractal time random walks, allows anomalous diffusion effects to be incorporated into both of these models with the very important result that one may determine how the decay constants of the normal diffusion that is the eigenvalues are affected by 
the anomalous behavior. Our results may be regarded as a generalization of the solution for the normal rotational Brownian motion [6] to a fractal time random walk Stosszahlansatz (giving rise to anomalous diffusion). We remark that just as the conventional Debye relaxation equation $(\sigma=1)$, the Cole-Cole equation may be derived from a number of very different models (see, e.g., Refs. [20-24]). However, the advantage of using kinetic equations (such as the fractional Fokker-Planck equation) over all other approaches is that the origin of such equations lies in the general theory of stochastic processes. Moreover, one may explicitly include both internal and external field potentials and exactly calculate their effect on the relaxation process. We also remark that in dielectric relaxation our results are restricted to the low frequency range, as defined by the inequality $\omega \tau_{\gamma}$ $\leqslant 1(\gamma=\|, \perp)$, because the theory does not include the effects of molecular inertia. A consistent treatment of inertial effects must be carried out using the appropriate inertial kinetic equation for the evolution of the probability density function in phase space [6].

\section{ACKNOWLEDGMENTS}

The support of this work by INTAS (Project No. 01-2341) and the TCD Trust is gratefully acknowledged.

\section{APPENDIX: MATRICES $\mathbf{Q}_{l}^{\gamma \pm}, \mathbf{Q}_{l}^{\gamma}$ AND INITIAL VALUE VECTORS C ${ }_{l}^{\gamma}(0)$}

The $2 \times 2$ matrices $\mathbf{Q}_{l}^{\gamma \pm}, \mathbf{Q}_{l}^{\gamma}$ in Eq. (27) are

$$
\begin{aligned}
& \mathbf{Q}_{l}^{\|-}=\left(\begin{array}{cc}
\frac{4 \xi_{V}(l-1)(2 l-1) l}{(4 l-1)(4 l-3)} & \frac{\xi(2 l-1) l}{4 l-1} \\
0 & \frac{2 \xi_{V}(2 l-1)(2 l+1) l}{(4 l+1)(4 l-1)}
\end{array}\right) \\
& \mathbf{Q}_{l}^{\|}=\left(\begin{array}{cc}
l(2 l-1)\left[\frac{2 \xi_{V}}{(4 l-3)(4 l+1)}-1\right] & \frac{-\xi l(2 l-1)}{4 l-1} \\
\frac{\xi l(2 l+1)}{4 l+1} & l(2 l+1)\left[\frac{2 \xi_{V}}{(4 l+3)(4 l-1)}-1\right]
\end{array}\right) \\
& \mathbf{Q}_{l}^{\|+}=\left(\begin{array}{cc}
\frac{-2 \xi_{V}(2 l+1)(2 l-1) l}{(4 l-1)(4 l+1)} & 0 \\
\frac{-\xi l(2 l+1)}{4 l+1} & \frac{-4 \xi_{V}(l+1)(2 l+1) l}{(4 l+1)(4 l+3)}
\end{array}\right) \\
& \mathbf{Q}_{l}^{\perp-}=\left(\begin{array}{cc}
\frac{4 \xi_{V} l^{2}(2 l-1)}{(4 l-1)(4 l-3)} & \frac{2 \xi l^{2}}{4 l-1} \\
0 & \frac{2 \xi_{V} l(2 l+1)^{2}}{(4 l-1)(4 l+1)}
\end{array}\right) \\
& \mathbf{Q}_{l}^{\perp}=\left(\begin{array}{cc}
\xi_{V} \frac{2 l(2 l-1)-3}{(4 l-3)(4 l+1)}-l(2 l-1) & -\frac{\xi(2 l-1)^{2}}{2(4 l-1)} \\
\frac{\xi(2 l+1)^{2}}{2(4 l+1)} & \xi_{V} \frac{2 l(2 l+1)-3}{(4 l-1)(4 l+3)}-l(2 l+1)
\end{array}\right) \\
& \mathbf{Q}_{l}^{\perp+}=\left(\begin{array}{cc}
-\frac{2 \xi_{V} l(2 l-1)^{2}}{(4 l-1)(4 l+1)} & 0 \\
-\frac{2 \xi l^{2}}{4 l+1} & -\frac{4 \xi_{V} l^{2}(2 l+1)}{(4 l+1)(4 l+3)}
\end{array}\right)
\end{aligned}
$$


The initial value vectors $\mathbf{C}_{l}^{\gamma}(0)$ in Eq. (31) may be determined using matrix continued fractions because the initial values $f_{l}^{\gamma}(0)$ are expressed just as in normal diffusion in terms of the equilibrium averages ([6], Chap. 8)

$$
\begin{gathered}
f_{l}^{\prime \prime}(0)=\xi_{1}\left[\left\langle\cos \vartheta P_{l+1}(\cos \vartheta)\right\rangle_{0}-\langle\cos \vartheta\rangle_{0}\left\langle P_{l}(\cos \vartheta)\right\rangle_{0}\right] \\
=\xi_{1}\left[\frac{l+1}{2 l+1}\left\langle P_{l+1}\right\rangle_{0}+\frac{l}{2 l+1}\left\langle P_{l-1}\right\rangle_{0}-\left\langle P_{1}\right\rangle_{0}\left\langle P_{l}\right\rangle_{0}\right], \\
f_{l}^{\perp}(0)=\xi_{1}\left\langle\cos ^{2} \varphi P_{1}^{1}(\cos \vartheta) P_{l}^{1}(\cos \vartheta)\right\rangle_{0} \\
=\xi_{1} \frac{l(l+1)}{2(2 l+1)}\left[\left\langle P_{l-1}\right\rangle_{0}-\left\langle P_{l+1}\right\rangle_{0}\right] .
\end{gathered}
$$

In turn, the equilibrium quantities $\left\langle P_{l}\right\rangle_{0}$ satisfy the set of equations ([6], Chap. 8)

$$
\mathbf{Q}_{l}^{\|-}\left(\begin{array}{c}
\left\langle P_{2 l-3}\right\rangle_{0} \\
\left\langle P_{2 l-2}\right\rangle_{0}
\end{array}\right)+\mathbf{Q}_{l}^{\|}\left(\begin{array}{c}
\left\langle P_{2 l-1}\right\rangle_{0} \\
\left\langle P_{2 l}\right\rangle_{0}
\end{array}\right)+\mathbf{Q}_{l}^{\|+}\left(\begin{array}{c}
\left\langle P_{2 l+1}\right\rangle_{0} \\
\left\langle P_{2 l+2}\right\rangle_{0}
\end{array}\right)=\mathbf{0} .
$$

The solution of Eq. (A3) is then given by

$$
\left(\begin{array}{c}
\left\langle P_{2 l-1}(\cos \vartheta)\right\rangle_{0} \\
\left\langle P_{2 l}(\cos \vartheta)\right\rangle_{0}
\end{array}\right)=\mathbf{S}_{l}^{\|}(0) \mathbf{S}_{l-1}^{\|}(0) \cdots \mathbf{S}_{1}^{\|}(0)\left(\begin{array}{l}
0 \\
1
\end{array}\right),
$$

where $\mathbf{S}_{l}^{\|}(0)=\boldsymbol{\Delta}_{l}^{\|}(0) \mathbf{Q}_{l}^{\|-}$and $\boldsymbol{\Delta}_{l}^{\|}(0)$ is given by Eq. (30) at $\boldsymbol{\omega}$ $=0$.

Thus according to Eqs. (A1), (A2), and (A4), the initial conditions $\mathbf{C}_{l}^{\gamma}(0)$ using matrix continued fractions are

$$
\begin{aligned}
\mathbf{C}_{l}^{\|}(0)= & \xi_{1}\left\{\left(\begin{array}{cc}
0 & 0 \\
\frac{2 l+1}{4 l+1} & 0
\end{array}\right) \mathbf{S}_{l+1}^{\|}(0)\right. \\
& \left.+\left(\begin{array}{cc}
-\left\langle P_{1}\right\rangle_{0} & \frac{2 l}{4 l-1} \\
\frac{2 l}{4 l+1} & -\left\langle P_{1}\right\rangle_{0}
\end{array}\right)\right] \mathbf{S}_{l}^{\|}(0) \\
& \left.+\left(\begin{array}{cc}
0 & \frac{2 l-1}{4 l-1} \\
0 & 0
\end{array}\right)\right\} \mathbf{S}_{l-1}^{\|}(0) \cdots \mathbf{S}_{1}^{\|}(0)\left(\begin{array}{l}
0 \\
1
\end{array}\right),
\end{aligned}
$$

$$
\begin{aligned}
\mathbf{C}_{l}^{\perp}(0)= & \xi_{1}\left\{\left[\begin{array}{cc}
0 & 0 \\
-\frac{l(2 l+1)}{4 l+1} & 0
\end{array}\right) \mathbf{S}_{l+1}^{\|}(0)\right. \\
& \left.+\left(\begin{array}{cc}
0 & -\frac{l(2 l-1)}{4 l-1} \\
\frac{l(2 l+1)}{4 l+1} & 0
\end{array}\right)\right] \mathbf{S}_{l}^{\|}(0) \\
& \left.+\left(\begin{array}{cc}
0 & \frac{l(2 l-1)}{4 l-1} \\
0 & 0
\end{array}\right)\right\} \mathbf{S}_{l-1}^{\|}(0) \cdots \mathbf{S}_{1}^{\|}(0)\left(\begin{array}{l}
0 \\
1
\end{array}\right) .
\end{aligned}
$$

[1] J. C. Dyre and N. B. Olsen, Phys. Rev. Lett. 91, 155703 (2003).

[2] K. S. Gilroy and W. A. Philips, Philos. Mag. B 43, 735 (1981).

[3] H. Fröhlich, Theory of Dielectrics, 2nd ed. (Oxford University Press, London, 1958).

[4] P. Debye, Polar Molecules (Chemical Catalog, New York, 1929) (Reprinted by Dover Publications, New York, 1954).

[5] W. T. Coffey, D. A. Garanin, and D. McCarthy, Adv. Chem. Phys. 117, 528 (2001).

[6] W. T. Coffey, Yu. P. Kalmykov and J. T. Waldron, The Langevin Equation, 2nd ed. (World Scientific, Singapore, 2004).

[7] P. Hänggi, P. Talkner, and M. Borkovec, Rev. Mod. Phys. 62, 251 (1990).

[8] P. G. Debenedetti and F. H. Stillinger, Nature (London) 410, 259 (2001).

[9] R. Mazo, Brownian Motion: Fluctuations, Dynamics and Applications (Oxford University Press, Oxford, 2002).

[10] W. T. Coffey, D. S. F. Crothers, D. Holland, and S. V. Titov, J. Mol. Liq. 114, 165 (2004).

[11] W. T. Coffey, J. Mol. Liq. 114, 5 (2004).

[12] P. Lunkenheimer, U. Schneider, R. Brandt, and A. Loidl, Contemp. Phys. 41, 15 (2000).

[13] G. P. Johari and M. Goldstein, J. Chem. Phys. 53, 2372 (1970).
[14] M. Paluch, C. M. Roland, S. Pawlus, J. Ziolo, and K. L. Ngai, Phys. Rev. Lett. 91, 115701 (2003).

[15] T. Blochowicz and E. A. Rössler, Phys. Rev. Lett. 92, 225701 (2004).

[16] A. Sanz, A. Nogales, T. A. Ezquerra, N. Lotti, and L. Finelli, Phys. Rev. E 70, 021502 (2004).

[17] E. W. Montroll and G. H. Weiss, J. Math. Phys. 6, 167 (1965); G. H. Weiss, Aspects and Applications of the Random Walk (North-Holland, Amsterdam, 1994).

[18] D. ben-Avraham and S. Havlin, Diffusion and Reactions in Fractals and Disordered Systems (Cambridge University Press, London, 2000).

[19] G. Williams and D. C. Watts, Trans. Faraday Soc. 66, 80 (1970).

[20] M. F. Shlesinger and E. W. Montroll, Proc. Natl. Acad. Sci. U.S.A. 81, 1280 (1984).

[21] W. G. Glöckle and T. F. Nonnenmacher, Macromolecules 24, 6426 (1991).

[22] W. T. Coffey, Yu. P. Kalmykov and S. V. Titov, J. Chem. Phys. 116, 6422 (2002).

[23] V. V. Novikov and V. P. Privalko, Phys. Rev. E 64, 031504 (2001).

[24] K. Weron and M. Kotulski, Physica A 232, 180 (1996).

[25] W. Paul and J. Baschnagel, Stochastic Processes from Physics 
to Finance (Springer-Verlag, Berlin, 1999).

[26] Yu. P. Kalmykov, W. T. Coffey, and S. V. Titov, Phys. Rev. E 69, 021105 (2004).

[27] R. Metzler and J. Klafter, Adv. Chem. Phys. 116, 223 (2001); Phys. Rep. 339, 1 (2000).

[28] E. Barkai, R. Metzler, and J. Klafter, Phys. Rev. E 61, 132 (2000).

[29] R. Metzler, E. Barkai, and J. Klafter, Phys. Rev. Lett. 82, 3563 (1999).

[30] S. G. Samko, A. A. Kilbas, and O. I. Marichev, Fractional Integrals and Derivatives, Theory and Applications (Gordon and Breach, New York, 1993).

[31] Handbook of Mathematical Functions, edited by M. Abramowitz and I. Stegun (Dover, New York, 1964).

[32] D. A. Garanin, Phys. Rev. E 54, 3250 (1996).

[33] A. Aharoni, Phys. Rev. 177, 793 (1969).

[34] W. F. Brown, Phys. Rev. 130, 1677 (1963); IEEE Trans. Magn. 15, 1196 (1979).

[35] W. Götze and L. Sjögren, Rep. Prog. Phys. 55, 241 (1992).

[36] M. Goldstein, J. Chem. Phys. 51, 3728 (1969).

[37] G. Power and J. K. Vij, J. Chem. Phys. 120, 5455 (2004).

[38] P. C. Fannin and A. T. Giannitsis, J. Mol. Liq. 114, 89 (2004). 